Check for updates

Cite this: RSC Adv., 2017, 7, 53048

\title{
Investigating the lithium ion battery electrolyte additive tris (2,2,2-trifluoroethyl) phosphite by gas chromatography with a flame ionization detector (GC-FID)
}

\author{
Tim Dagger, ${ }^{\text {ab }}$ Jonas Henschel, ${ }^{\text {ab }}$ Babak Rad, ${ }^{c}$ Constantin Lürenbaum, ${ }^{a}$ \\ Falko M. Schappacher, ${ }^{a}$ Martin Winter ${ }^{\mathrm{abc}}$ and Sascha Nowak (DD *a
}

\begin{abstract}
The quantification of lithium ion battery electrolyte additives provides challenges in terms of methods and instrumentation. In this work, the detectability of the flame retardant additive tris(2,2,2-trifluoroethyl) phosphite (TTFPi) differs unusually when added to a standard electrolyte ( $1 \mathrm{M} \mathrm{LiPF}_{6}$ in ethylene carbonate (EC) : dimethyl carbonate (DMC) $1: 1$ wt\%) using gas chromatography with a flame ionization detector (GC-FID). In this work, nuclear magnetic resonance (NMR), ion trap time of flight mass spectrometry (IT-TOFTM MS) and gas chromatography-mass spectrometry (GC-MS) are used to investigate a pure TTFPi solution and a standard battery electrolyte with TTFPi as an additive with regard to parasitic TTFPi consuming reactions and different TTFPi concentrations, respectively. NMR and IT-TOFTM MS measurements confirm the chemical stability of the TTFPi/standard electrolyte mixture and concentration dependent GC-MS and GC-FID experiments indicate a premature FID saturation limit for TTFPi in presence of standard electrolyte. The findings explain the counterintuitive absence of TTFPi for higher concentrations and provide important information for future sample preparation.
\end{abstract}

Received 26th August 2017 Accepted 11th November 2017

DOI: $10.1039 / c 7 r a 09476 k$

rsc.li/rsc-advances carbonates; ${ }^{5,6}$ in the present example: $1 \mathrm{M} \mathrm{LiPF}_{6}$ in ethylene carbonate (EC) : dimethyl carbonate (DMC) 1:1 wt\%, LP30. The flammability of the electrolyte still offers room for further improvement in LIB safety. The usage of electrolyte additives that lower the electrolyte's flammability is a cost efficient strategy to improve LIB safety characteristics. Phosphorous agents are frequently used as flame retardant additives. Among them, the phosphites are used as functional electrolyte additives and can fulfill various tasks. Typical functions attributed to phosphites are the improvement of the capacity retention during cycling with elevated cut-off voltages, ${ }^{7-11}$ improvement of the thermal stability ${ }^{12}$ and the improvement of the cell safety due to reduction of the electrolyte's flammability.,13-16

Nevertheless, phosphites applied in the standard electrolyte are likely to decompose due to oxidation on the cathode during prolonged cycling..$^{\mathbf{8 1 0}}$ They are even known to be consumed during storage due to spontaneous reactions with $\operatorname{LiPF}_{6} \cdot{ }^{17}$ The flame retardant additive tris(2,2,2-trifluoroethyl) phosphite (TTFPi) was investigated towards its electrochemical performance in LIBs via gas chromatography methods, which are the commonly applied analytical techniques for this kind of investigations. ${ }^{18,19}$ However, post-mortem investigations of the TTFPi electrolyte that were applied in LIB cells revealed that the additive was not detectable during GC-FID measurements even though it could be easily detected by GC-MS experiments. In order to understand this phenomenon, GC-MS and GC-FID
${ }^{a}$ MEET Battery Research Center, Westfälische Wilhelms-Universität Münster Corrensstr. 46, D-48149 Münster, Germany. E-mail: sascha.nowak@uni-muenster. de; Fax: +49 25183 36032; Tel: +492518336735

${ }^{b}$ Institute of Physical Chemistry, Westfälische Wilhelms-Universität Münster, Corrensstr. 28/30, D-48149 Münster, Germany

'Helmholtz Institute Münster, IEK-12, Research Center Jülich GmbH, Corrensstr. 46, D48149 Münster, Germany 
measurements with pure TTFPi and 5 wt\% TTFPi in the standard LP30 electrolyte were repeated and deeply investigated. Fig. 1 displays the chromatograms of the same mixture of TTFPi in the LP30 electrolyte measured with GC-MS in Fig. 1a and with GC-FID in Fig. 1b. It is clearly observable, that the TTFPi is not detectable via GC-FID. However, pure TTFPi is detectable in GCMS as well as in GC-FID (Fig. 1c and d).

The findings serve as starting point and give the rise to the question why TTFPi cannot be detected in the GC-FID measurement after added to LP30. Therefore two hypotheses can be proposed:

(1) The molecule decomposes after addition to LP30. Apparent decomposition products reveal similar spectra to TTFPi and are therefore difficult to distinguish from TTFPi by GC-MS. Nevertheless, apparent decomposition is clearly visible in GC-FID.

(2) The molecule remains stable, but is harder to detect by GC-FID when added to LP30, indicating a severe matrix dependency. Therefore, samples of TTFPi in LP30 are easy to detect via GC-MS, but almost not visible during GC-FID experiments.

The first hypothesis is investigated by a comparison of the pure TTFPi with TTFPi in LP30 via NMR and high-resolution MS. Both methods are suitable and have been applied for the analysis of electrolyte constituents. ${ }^{20-23}$ Decomposition products in the solution of TTFPi with LP30 would indicate parasitic reactions of the additive and the electrolyte and thus confirm hypothesis 1 .

The second hypothesis is reviewed with the help of GC-MS and GC-FID measurements using different concentrations of
TTFPi. Since a diluted sample is not detectable, a more concentrated solution of TTFPi and LP30 would provide a TTFPi signal even in the GC-FID experiments.

\section{Experimental}

Two solutions of (I) pure TTFPi and (II) $5 \mathrm{wt} \%$ TTFPI in LP30 were investigated. Therefore, LP30 (1 M LiPF 6 in ethylene carbonate (EC) : dimethyl carbonate (DMC) $1: 1 \mathrm{wt} \%$ ) (battery grade, BASF) was mixed with 5 wt $\%$ TTFPi ( $>98.5 \%$, Sigma Aldrich). Samples were diluted with acetonitrile (99.9\% VWR) according to Table 1.

Gas chromatography-mass spectrometry (GC-MS) measurements were performed using a Perkin Elmer GC-MS Clarus 600 including a Restek Rxi-5Sil MS column $(30 \mathrm{~m} \times 0.25 \mathrm{~mm} \times$ $0.25 \mu \mathrm{m})$ with related TurboMass 5.4.2- and Clarity 7.3.0.0.95 software. At $200{ }^{\circ} \mathrm{C}$ injector temperature, $1 \mu \mathrm{L}$ of the diluted sample was injected to the injector. Helium with a constant

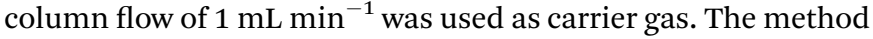
used a split of $1: 20$ and the following oven program: the initial temperature of $40{ }^{\circ} \mathrm{C}$ was held for $3 \mathrm{~min}$, followed by a first ramp with a rate of $12{ }^{\circ} \mathrm{C} \min ^{-1}$ up to $85^{\circ} \mathrm{C}$, and a second ramp with $30{ }^{\circ} \mathrm{C} \min ^{-1}$ up to $200{ }^{\circ} \mathrm{C}$ (held for $1 \mathrm{~min}$ ). Electron ionization with an ion source temperature of $250{ }^{\circ} \mathrm{C}$ and a detector voltage of $350 \mathrm{~V}$ at a filament voltage of $70 \mathrm{eV}$ was applied. Recorded masses were in between $m / z=30-600$.

The Perkin Elmer equipment offers two injectors. Therefore, quantitative experiments with detection via flame ionization (GC-FID) were performed using the same Perkin Elmer GC-MS Clarus 600 Chromatograph and a nonpolar Restek Rxi-5ms
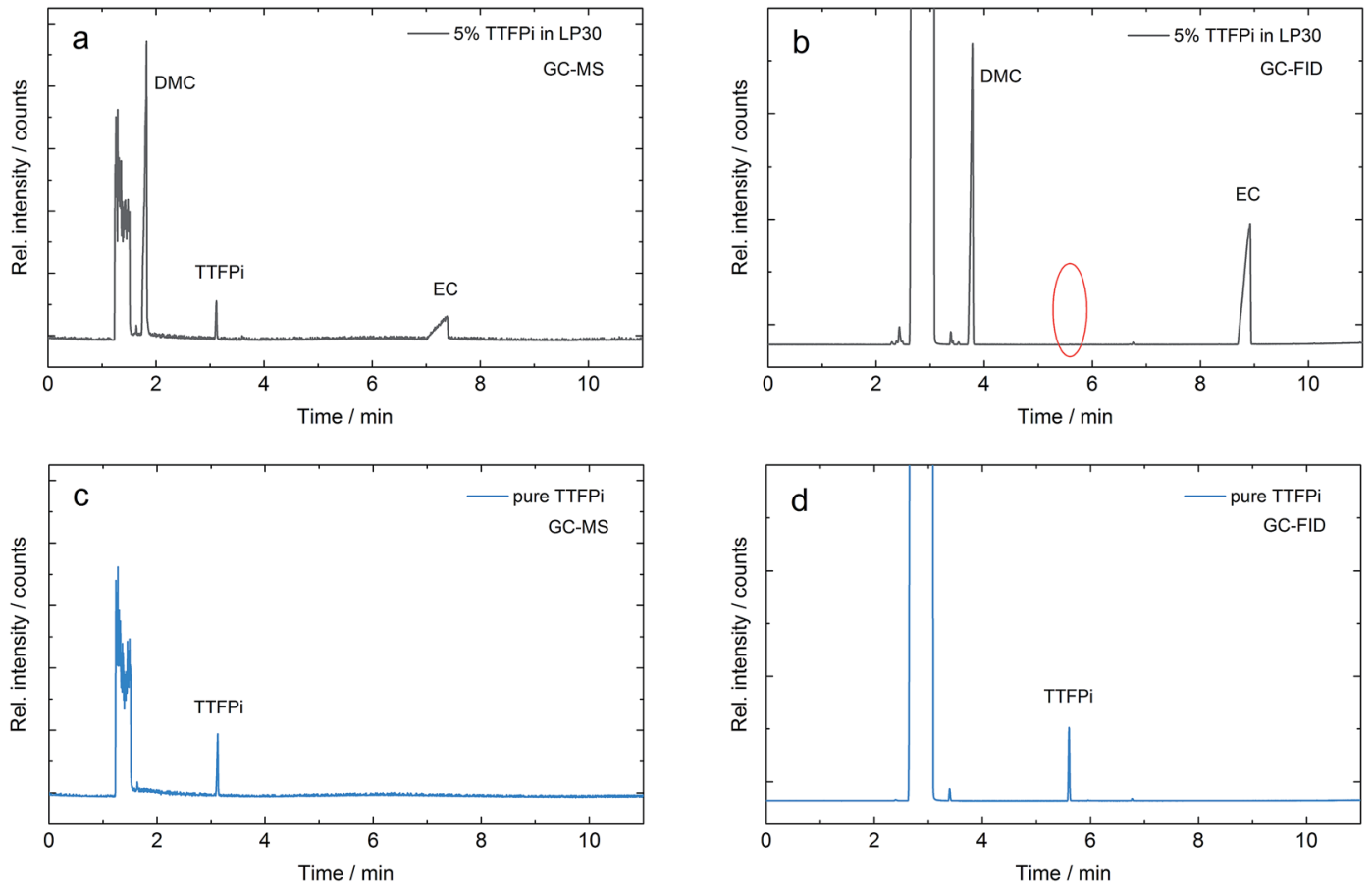

Fig. 1 (a) GC-MS and (b) GC-FID measurements of the same mixture of 5 wt\% TTFPi in LP30. (c) GC-MS and (d) GC-FID measurement of pure TTFPi. Samples are diluted $(1: 50)$ with acetonitrile. The missing TTFPi signal is indicated by a red circle. 
Table 1 Target- and practical TTFPi concentrations of pure TTFPi and 5 wt\% TTFPi in LP30 diluted with acetonitrile

\begin{tabular}{llll}
\hline Dilution number & $\begin{array}{l}\text { Target TTFPi concentrations/ } \\
\mathrm{mg} \mathrm{g}^{-1}\end{array}$ & $\begin{array}{l}\text { Actual TTFPi concentrations } \\
\text { sd pure TTFPi/mg g }\end{array}$ & $\begin{array}{l}\text { Actual TTFPi concentrations } \\
\text { sd 5 wt\% TTFPi in LP30/mg g }\end{array}$ \\
\hline 1 & 0.25 & 0.19 & 0.28 \\
2 & 0.75 & 0.77 & 0.71 \\
3 & 1.25 & 1.53 & 1.04 \\
4 & 2.00 & 2.33 & 2.03 \\
5 & 3.00 & 3.12 & 2.67 \\
\end{tabular}

column $(30 \mathrm{~m} \times 0.25 \mathrm{~mm} \times 0.25 \mu \mathrm{m})$. Software, temperature program and measurement properties were adapted from GC-MS measurements.

Nuclear Magnetic Resonance (NMR) experiments were performed using a Bruker Avance III $400\left(400.03 \mathrm{MHz},{ }^{1} \mathrm{H}\right.$; $376.37 \mathrm{MHz},{ }^{19} \mathrm{~F}$; $161.93 \mathrm{MHz},{ }^{31} \mathrm{P}$ ) spectrometer. All experiments were performed at $26.85{ }^{\circ} \mathrm{C}$ and were internally referenced to residual solvent resonances. Chemical shifts $(\delta)$ were reported in parts per million (ppm). Pure TTFPi and $5 \mathrm{wt} \%$
TTFPi in LP30 were diluted with pre-dried deuterated benzene $\left(\mathrm{C}_{6} \mathrm{D}_{6}\right.$; water content less than $\left.10 \mathrm{ppm}\right)$ in an argon filled glovebox. For the purity check of TTFPi, $50 \mathrm{mg}$ of TTFPi were dissolved in $600 \mu \mathrm{L} \mathrm{C}_{6} \mathrm{D}_{6}$. A J. Young NMR tube has been used for NMR experiments to avoid any contact with air. In order to analyze the chemical reactivity of TTFPi with LP30 and avoid any side reaction between the residual amount of HF (originated from $\mathrm{LiPF}_{6}$ in LP30) and glass, a fluorinated ethylene propylene (FEP) NMR tube ( $3 \mathrm{~mm} \varnothing$, Wilmad-labglass) were
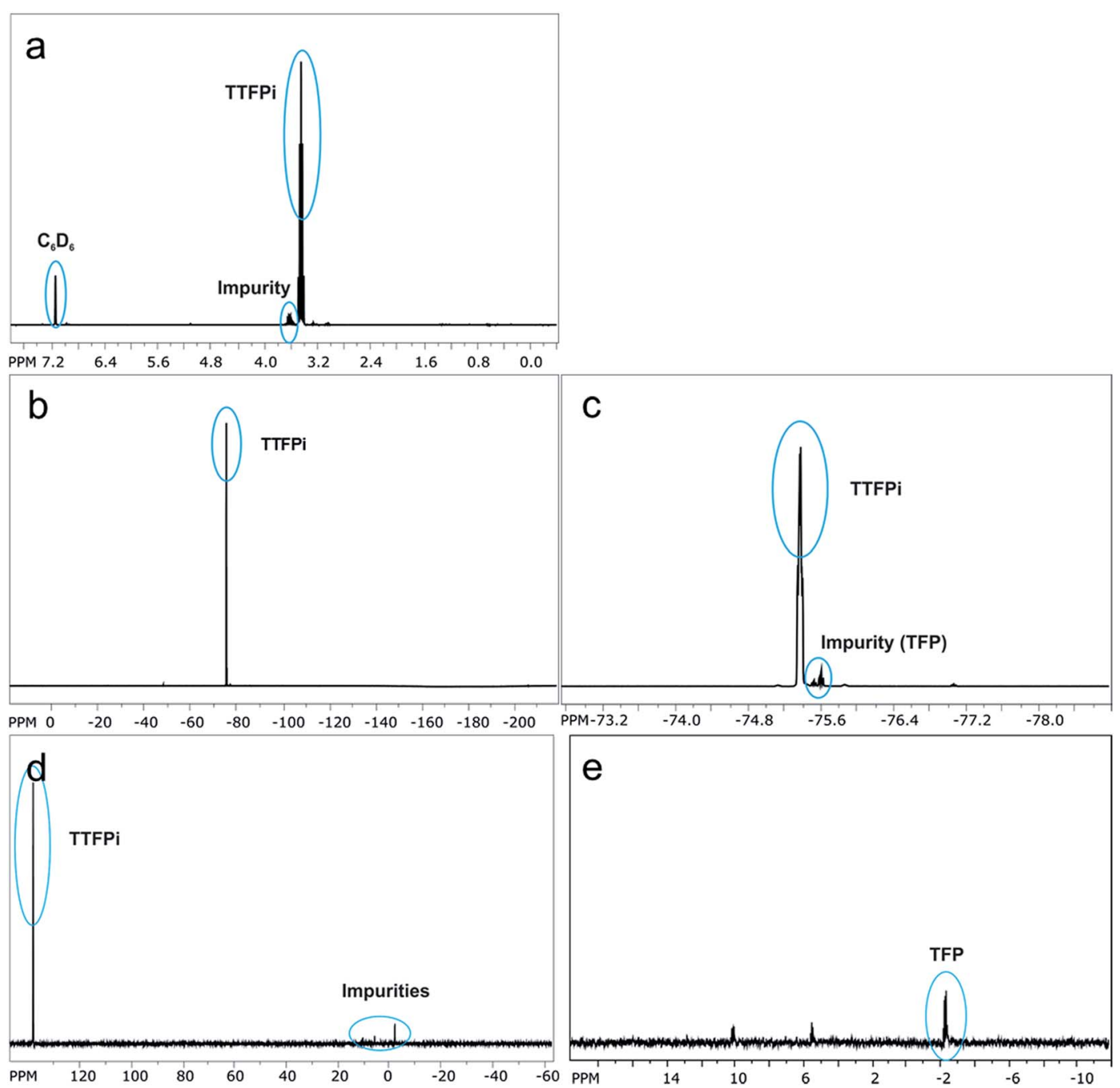

Fig. 2 (a) ${ }^{1} \mathrm{H}$ NMR-spectrum of pure TTFPi, (b) ${ }^{19} \mathrm{~F}$ NMR-spectrum of pure TTFPi, (c) zoom of ${ }^{19} \mathrm{~F}$ NMR-spectrum of pure TTFPi. (d) ${ }^{31} \mathrm{P}$ NMRspectrum of pure TTFPi. (e) Zoom of ${ }^{31} \mathrm{P}$ NMR-spectrum of pure TTFPi. 
used. The FEP tube containing $300 \mu \mathrm{L}$ of sample was closed by a polytetrafluorethylene (PTFE) plug and placed in a J-Young NMR tube $(5 \mathrm{~mm} \varnothing)$ containing $100 \mu \mathrm{L}$ of benzene- $\mathrm{d}_{6}\left(\mathrm{C}_{6} \mathrm{D}_{6}\right.$, 99.6 at\% D, Sigma Aldrich). In this way, any contact between the deuterated solvent and electrolyte mixtures, which could produce unwanted products, was prevented.

For qualitative investigation of pure TTFPi and TTFPi in contact with LP30, the LCMS-IT-TOF ${ }^{\text {TM }}$ from Shimadzu (Kyoto, Japan) in the direct injection mode was used. Therefore, $1 \mu \mathrm{L}$ sample was injected by the UHPLC Nexera X2 SIL-30AC auto sampler. It was directed to the hybrid ion trap time of light mass spectrometer (IT-TOF ${ }^{\text {TM }}$ MS) with a flow rate of $0.2 \mathrm{~mL} \mathrm{~min} \mathrm{~m}^{-1}$ ( $80: 20$ acetonitrile/water). The ionization was performed with electrospray ionization in the positive mode at $4.5 \mathrm{kV}$. The curved desolvation line temperature was $230{ }^{\circ} \mathrm{C}$, the heat block temperature $230{ }^{\circ} \mathrm{C}$ and the nebulizing gas flow was set to 1.5 $\mathrm{L} \mathrm{min}^{-1}$. The pressure of the nitrogen drying gas was $97 \mathrm{kPa}$, the detector voltage at $1.67 \mathrm{kV}$. The mass range was set from 150-2000 Da. For confirmation of the structure, $\mathrm{MS}^{4}$ experiments were performed. The collision induced dissociation energy (CID) was optimized for every $\mathrm{MS}^{n}$ stage.

\section{Results and discussion}

\subsection{Nuclear magnetic resonance}

In order to investigate whether the TTFPi is chemical stable in LP30, and thereby question hypothesis 1, long-term NMR experiments were performed. The findings revealed, that pure TTFPi (>98.5\%, Sigma Aldrich) contains some impurities of trifluoroethyl phosphate (TFP) (Fig. 2). Further, the
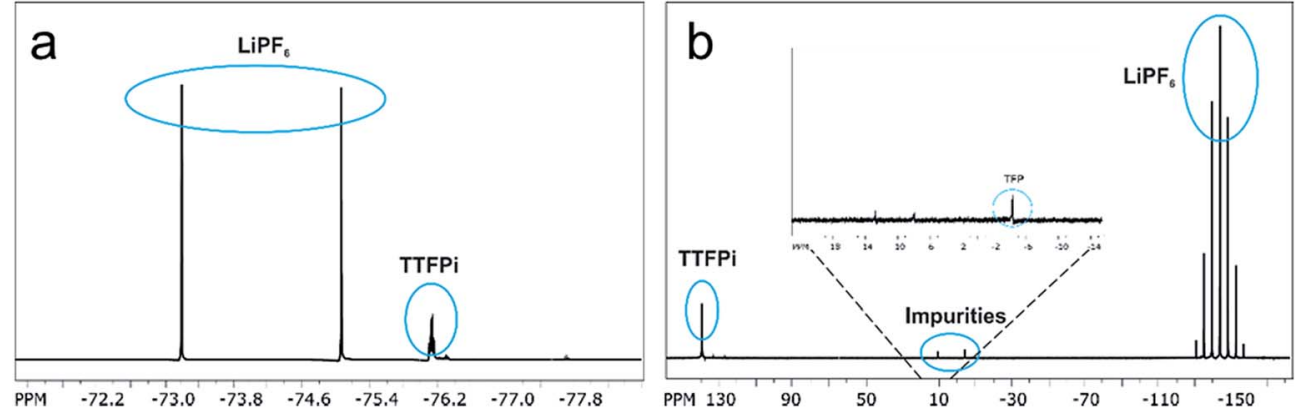

Fig. 3 NMR-spectra of the electrolyte containing 5 wt\% additive investigated six weeks after formulation. (a) ${ }^{19} \mathrm{~F} \mathrm{NMR-spectrum} \mathrm{of} \mathrm{TTFPi.} \mathrm{(b)}{ }^{31} \mathrm{P}$ NMR-spectrum of TTFPi.
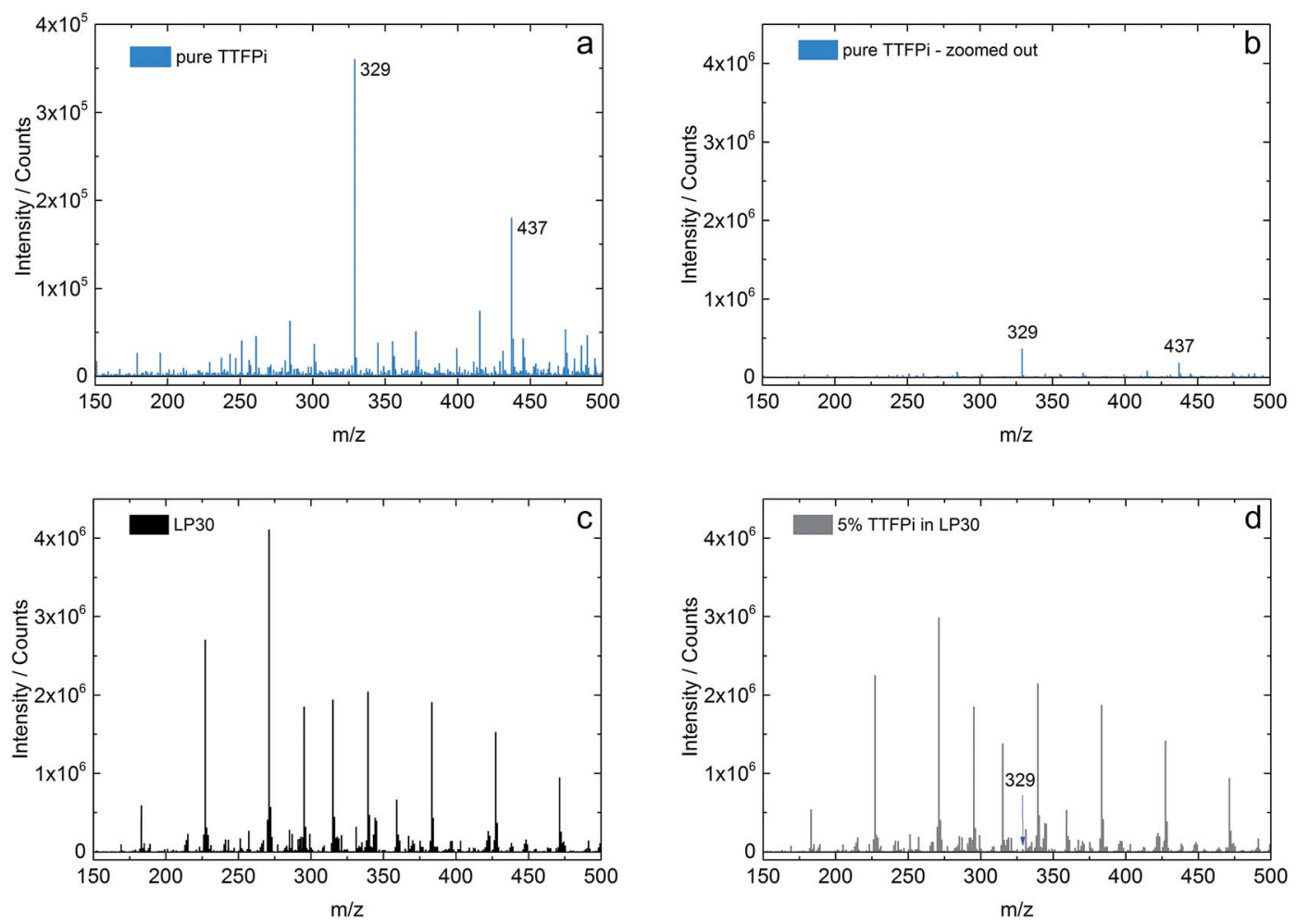

Fig. 4 Mass spectra of pure TTFPi, LP30 and 5 wt\% TTFPi in LP30 measured with IT-TOFTM MS. 
measurements prove that TTFPi is stable in mixture with LP30. No decomposition products could be observed after three weeks of storage under inert atmosphere (Fig. 3).

\subsection{Ion trap time of flight mass spectrometry}

In order to investigate the stability of the TTFPi in LP30, solutions of pure TTFPi, pure LP30 and of a mixture of $5 \mathrm{wt} \%$ TTFPi with LP30 are measured using an ion trap of flight mass spectrometer (IT-TOF MS). The results of the single solutions can be compared with the mixture of TTFPi and LP30. Fig. 4a and b display the spectra of pure TTFPi. Fig. $4 \mathrm{~b}$ is zoomed out, so that a wider scaling of the $Y$-axis is presented. The scale of Fig. $4 \mathrm{~b}$ displays the same scale that is used in Fig. $4 \mathrm{c}$ and $\mathrm{d}$ for reasons of comparability. Fig. 4c shows the spectrum of LP30 and Fig. 4d presents a spectrum of $5 \mathrm{wt} \%$ TTFPi in LP30. The spectrum of TTFPi (Fig. 4a) shows a signal for the $\mathrm{m} / \mathrm{z}$ value of 328.9963 (intensity of 360000 counts), which can be clearly attributed to TTFPi (molecular mass of 327.9911). In addition, a $m / z$ value of 437.1945 (180 000 counts) is visible. By comparison of the spectrum of LP30, which is depicted in Fig. 4c, with the spectrum of TTFPi mixed with LP30 depicted in Fig. 4d, just one additional signal is observable. This peak shows the $\mathrm{m} / \mathrm{z}$ value of 328.9991 and can be attributed to TTFPi (mass deviation $2.13 \mathrm{ppm}$ ). The intensity of this signal (33000 counts) is in accordance to the dilution of TTFPi in LP30. The Fig. 4b$\mathrm{d}$ provide the same scaling of the $Y$-axis, so that the intensities are roughly comparable. Besides the signal for TTFPi, there were no additional signals found in the spectra of Fig. 4d. This is an indication that no TTFPi decomposition products are formed. Therefore, it can be concluded that TTFPi is stable in LP30 according to the IT-TOF ${ }^{\mathrm{TM}}$ MS results.

In order to specifically confirm the structure of the TTFPi, $\mathrm{MS}^{4}$ experiments are performed. The results of the $\mathrm{MS}^{4}$ fragmentation are presented in Fig. 5. With a $m / z$ value of 328.9963

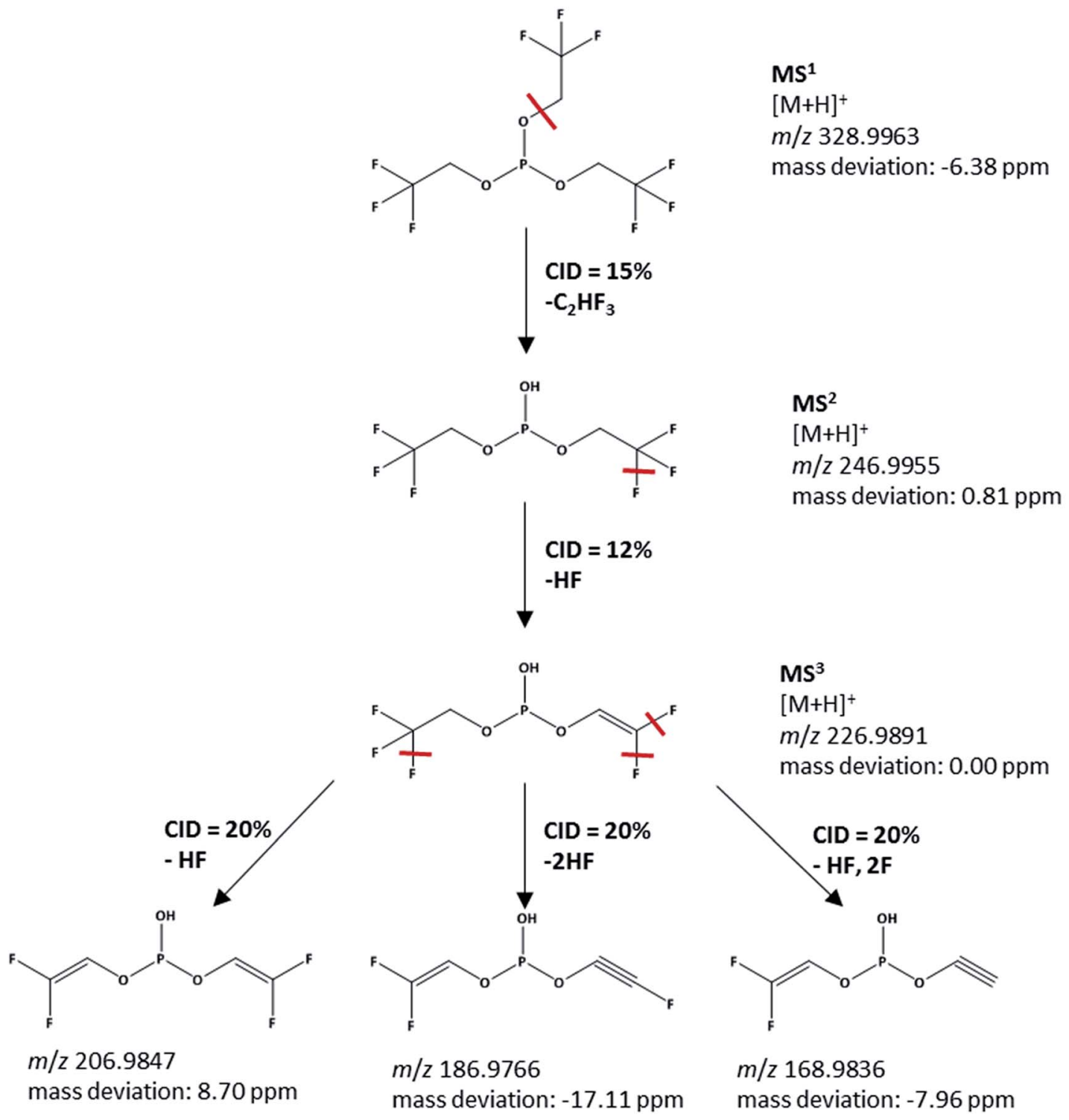

$\mathrm{MS}^{4}$

$[\mathrm{M}+\mathrm{H}]^{+}$

Fig. 5 MS $^{4}$ fragmentation pattern of pure TTFPi measured with IT-TOFTM MS. 

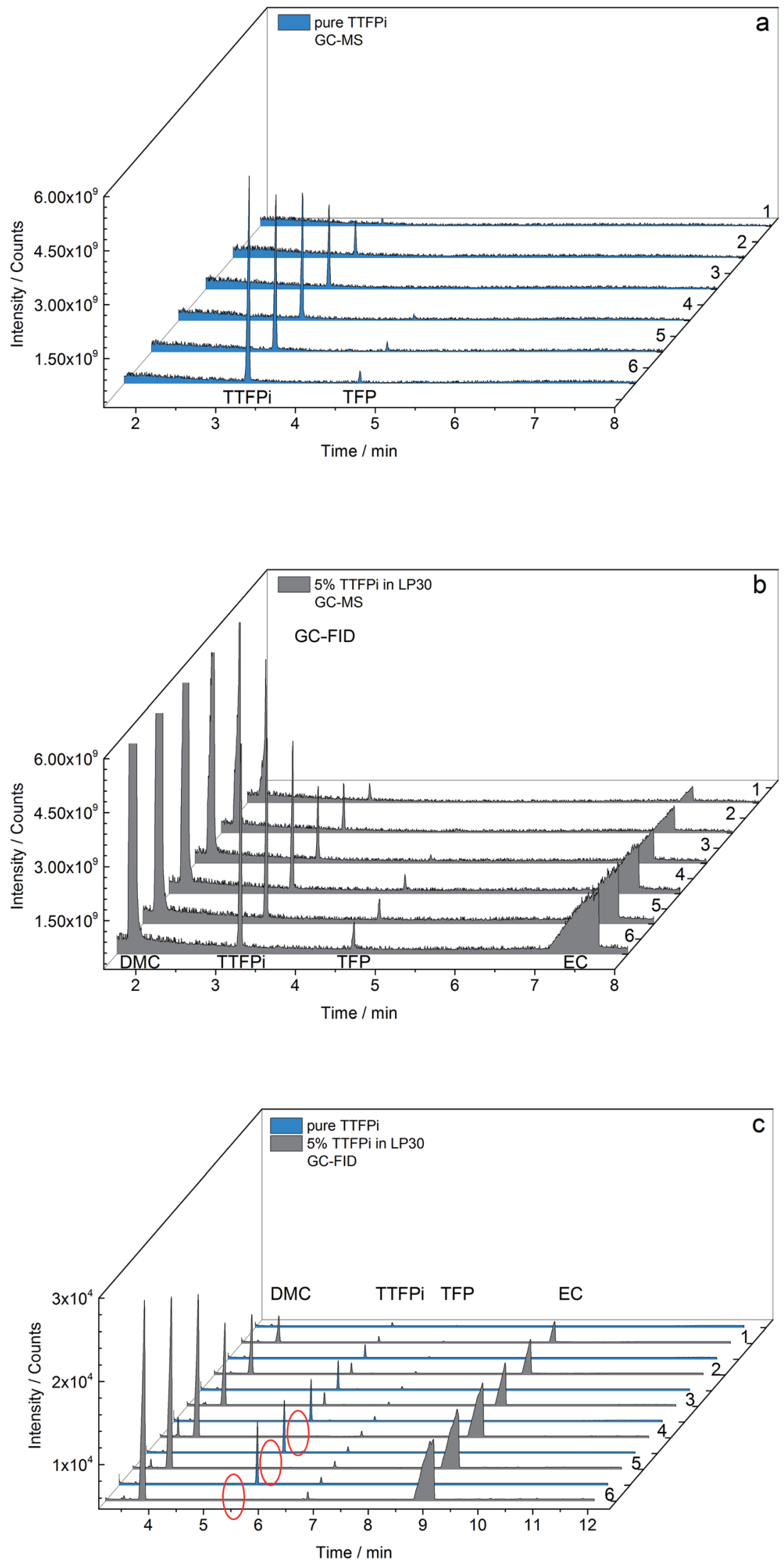

Fig. 6 Results of concentration depending ( $a$ and b) GC-MS and (c) GC-FID measurements of pure TTFPi and 5 wt\% TTFPi in LP30. Red circles indicate missing TTFPi peaks for higher concentrations. 
for pure TTFPi, the signal in Fig. $4 d(\mathrm{~m} / \mathrm{z}$ value of 328.9991$)$ can be clearly attributed to TTFPi and not to a decomposition product with a similar spectrum as proposed in hypothesis 1 . The IT-TOF ${ }^{\mathrm{TM}}$ MS results therefore confirm the NMR results in terms of the TTFPi stability in LP30. TTFPi is stable in LP30 and does not decompose. Consequently, it can be concluded, that hypothesis 1 is not valid:

(1) The molecule decomposes after addition of LP30. Apparent decomposition products reveal similar spectra to TTFPi and are therefore difficult to distinguish from TTFPi by GC-MS. Nevertheless, the apparent decomposition process is clearly visible in the GC-FID results.

\subsection{Concentration dependence of GC-MS and GC-FID measurements in comparison}

In order to investigate whether according to hypothesis 2 a more concentrated solution of TTFPi and LP30 would provide a TTFPi signal even in the GC-FID experiments, two serial dilutions (sd) of TTFPi are investigated (Table 1).

(2) The molecule remains stable, but is harder to detect by GC-FID when mixed with LP30, indicating a severe matrix dependency. Therefore, samples of TTFPi in LP30 are easy to detect via GC-MS, but almost not visible during GC-FID experiments.

The first sd includes 6 samples of pure TTFPi diluted in acetonitrile. The second sd includes 6 samples of $5 \mathrm{wt} \%$ TTFPi in LP30 diluted in acetonitrile. Both sd show comparable TTFPi amounts for each dilution of the series. Table 1 summarizes the target TTFPi concentrations of both sd, the actual concentration of the pure TTFPi sd and the actual TTFPi concentration of the sd of the $5 \mathrm{wt} \%$ TTFPi in LP30 mixture. It can be seen, that the TTFPi concentrations of both sd are comparable.

Fig. 6 combines the GC-MS and GC-FID results of the sd of pure TTFPi and TTFPi in mixture with LP30. The GC-MS measurements depicted in Fig. 6a show the decreasing TTFPi concentration in the samples 6 to 1 of diluted pure TTFPi. Even the smallest concentration of $0.19 \mathrm{mg} \mathrm{g}^{-1}$ is recognizable. With increasing TTFPi (3.2 $\mathrm{min})$ concentrations of the dilution number 4, 5 and 6 , the TFP ( $4.7 \mathrm{~min}$ ) impurities appear clearer. The TFP impurities could also be detected via NMR measurements before. Regarding Fig. 6b, additional signals for DMC and EC can be attributed to the LP30. Furthermore, the decreasing concentration of TTFPi, TFP and EC from dilution number 6 to sample 1 is visible. In addition, the TFP signal is already observable in dilution number 3 , even though the TTFPi concentration in dilution number 3 of the sd of TTFPi in LP30 is lower in comparison to the TTFPi concentration of the pure TTFPi sd (1.04 $\mathrm{mg} \mathrm{g}^{-1}$ compared to $1.53 \mathrm{mg} \mathrm{g}^{-1}$ ). However, in both GC-MS results Fig. $6 \mathrm{a}$ and $\mathrm{b}$ the decreasing TTFPi concentration is visible.

The results of the GC-FID measurements of both sd are shown together in Fig. 6c. Just like the GC-MS results, the GCFID results of the pure TTFPi (blue) show a consistently decreasing TTFPi concentration. The TFP is already detectible in dilution number 1 , which is two dilutions prior to the GC-MS measurements. Due to the lower LOD of the GC-FID measurement results appear more sensitive for TFP. The biggest difference between the results of the GC-MS and the GCFID results is that the TTFPi concentration do not increase from dilution number 1 to dilution number 6. The TTFPi concentration just increases up to dilution number 3 . Samples with the dilution numbers 4 to 6 which contained initially $\approx 2 \mathrm{mg} \mathrm{g}^{-1}$ to $\approx 3 \mathrm{mg} \mathrm{g}^{-1}$ TTFPi show no TTFPi signal.

Hypothesis 2 can partly be confirmed. TTFPi as flame retardant additive is in general hard to detect via FID. The molecule serves just six carbon atoms and is therefore hard to ionize. Especially in combination with the carbonates of the LP30 LIB electrolyte, the saturation point of TTFPi is reached early (between $1 \mathrm{mg} \mathrm{g}^{-1}$ and $2 \mathrm{mg} \mathrm{g}^{-1}$ ). Therefore, a higher concentration of TTFPi in mixture with LP30 does not provide increased TTFPi signals in the GC-FID measurements. A reason for that could be a lack of the TTFPi volatility that develop from methylation and ethylation of the TTFPi with linear carbonates during high temperature within the detector. Counterintuitively, a higher concentrated TTFPi LP30 mixture results in absence of TTFPi signals due to GC-FID saturation. However, flame extinguishment was reported for similar compounds which could hint on a likewise mechanism. ${ }^{24}$

The findings are of fundamental importance, since it is common practice to dilute GC-MS and GC-FID samples $\approx 1 / 100$ (ref. 25 and 26 ) or $\approx 1 / 50$ with acetonitrile. A dilution of $1: 100$ of $5 \mathrm{wt} \%$ TTFPi in LP30 would result in an overall concentration of $\approx 0.65 \mathrm{mg} \mathrm{g}^{-1}$ TTFPi which is below the saturation point. A dilution of $1: 50$ of $5 \mathrm{wt} \%$ TTFPi in LP30 with acetonitrile however, would result in an overall concentration of $\approx 1.3 \mathrm{mg}$ $\mathrm{g}^{-1}$ TTFPi and might exceed the saturation limit. All in all, the same electrolyte which contains 5 wt $\%$ of TTFPi would show different results in the GC-FID measurements depending on its dilution with acetonitrile.

\section{Conclusion}

Within this study it was shown that the flame retardant electrolyte additive tris (2,2,2-trifluoroethyl) phosphite (TTFPi) provided a GC-FID saturation limit when combined with an organic carbonate solvent based electrolyte. The hypothesis that the TTFPi decomposes in presence of LP30 could be disproved by NMR and IT-TOF ${ }^{\mathrm{TM}}$ MS. The hypothesis that TTFPi remains stable, but is less detectable in presence of LP30 could be partly confirmed. An increased TTFPi-LP30 (1 M LiPF 6 in ethylene carbonate (EC) : dimethyl carbonate (DMC) $1: 1 \mathrm{wt} \%$ ) mixture resulted in the absence of TTFPi signals due to GC-FID saturation. Added to LP30 and acetonitrile, the saturation point of TTFPi for the FID was between $1 \mathrm{mg} \mathrm{g}^{-1}$ and $2 \mathrm{mg} \mathrm{g}^{-1}$. The results revealed that the sample preparation and sample dilution degree highly influenced the outcome of GC-FID TTFPi investigation. This finding is of great importance for postmortem analysis of lithium ion battery electrolytes in which TTFPi are applied. Therefore, it is concluded that GC-FID measurements of fluorinated phosphites should be conducted in combination with further techniques like GC-MS. Further option are displayed by GC in combination with an electron capture detector (ECD) or a flame photometric detector (FPD). 
Future investigation could target the exact GC-FID TTFPi saturation limit of 5\% TTFPi in LP30. Further it would be interesting to investigate the GC-FID saturation limit of pure TTFPi as well as the saturation limit of TTFPi in dependence of the LP30 concentration.

\section{Conflicts of interest}

There are no conflicts to declare.

\section{Acknowledgements}

The authors would like to thank the German Federal Ministry of Education and Research (BMBF) for financial support. This work was conducted within the context of the project "Elektrolytlabor 4e" (03X4632). Marco Fritzen is acknowledged for help during sample preparation.

\section{References}

1 R. Wagner, N. Preschitschek, S. Passerini, J. Leker and M. Winter, J. Appl. Electrochem., 2013, 43, 481-496.

2 T. Placke, R. Kloepsch, S. Dühnen and M. Winter, J. Solid State Electrochem., 2017, 1-26.

3 P. Meister, H. Jia, J. Li, R. Kloepsch, M. Winter and T. Placke, Chem. Mater., 2016, 28, 7203-7217.

4 Z. J. Zhang, P. Ramadass and W. Fang, Lithium-Ion Batteries-Advances and Applications, ed. G. Pistoia, 2014, pp. 409-435.

5 R. W. Schmitz, P. Murmann, R. Schmitz, R. Müller, L. Krämer, J. Kasnatscheew, P. Isken, P. Niehoff, S. Nowak and G.-V. Röschenthaler, Prog. Solid State Chem., 2014, 42, 65-84.

6 C. Korepp, H. Santner, T. Fujii, M. Ue, J. Besenhard, K.-C. Möller and M. Winter, J. Power Sources, 2006, 158, 578-582.

7 N. von Aspern, S. Röser, B. Rezaei Rad, P. Murmann, B. Streipert, X. Mönnighoff, S. D. Tillmann, M. Shevchuk, O. Stubbmann-Kazakova, G.-V. Röschenthaler, S. Nowak, M. Winter and I. Cekic-Laskovic, J. Fluorine Chem., 2017, 198, 24-33.

8 S. Mai, M. Xu, X. Liao, J. Hu, H. Lin, L. Xing, Y. Liao, X. Li and W. Li, Electrochim. Acta, 2014, 147, 565-571.
9 Z. Li, Y. Zhang, H. Xiang, X. Ma, Q. Yuan, Q. Wang and C. Chen, J. Power Sources, 2013, 240, 471-475.

10 J. Pires, A. Castets, L. Timperman, J. Santos-Peña, E. Dumont, S. Levasseur, C. Tessier, R. Dedryvère and M. Anouti, J. Power Sources, 2015, 296, 413-425.

11 Y.-M. Song, J.-G. Han, S. Park, K. T. Lee and N.-S. Choi, J. Mater. Chem. A, 2014, 2, 9506-9513.

12 H. Xu, S. Xie, Q. Wang, X. Yao, Q. Wang and C. Chen, Electrochim. Acta, 2006, 52, 636-642.

13 X. Yao, S. Xie, C. Chen, Q. Wang, J. Sun, Y. Li and S. Lu, J. Power Sources, 2005, 144, 170-175.

14 N. D. Nam, I. J. Park and J.-G. Kim, ECS Trans., 2011, 33, 7-15.

15 T.-H. Nam, E.-G. Shim, J.-G. Kim, H.-S. Kim and S.-I. Moon, J. Power Sources, 2008, 180, 561-567.

16 S. Zhang, K. Xu and T. Jow, J. Power Sources, 2003, 113, 166172.

17 X. Qi, L. Tao, H. Hahn, C. Schultz, D. R. Gallus, X. Cao, S. Nowak, S. Röser, J. Li and I. Cekic-Laskovic, RSC Adv., 2016, 6, 38342-38349.

18 P. Murmann, X. Monnighoff, N. von Aspern, P. Janssen, N. Kalinovich, M. Shevchuk, O. Kazakova, G. V. Roschenthaler, I. Cekic-Laskovic and M. Winter, J. Electrochem. Soc., 2016, 163, A751.

19 X. Mönnighoff, P. Murmann, W. Weber, M. Winter and S. Nowak, Electrochim. Acta, 2017, 246, 1042-1051.

20 C. Schultz, S. Vedder, M. Winter and S. Nowak, Anal. Chem., 2016, 88, 11160-11168.

21 S. Wiemers-Meyer, M. Winter and S. Nowak, Phys. Chem. Chem. Phys., 2016, 18, 26595-26601.

22 S. Wiemers-Meyer, M. Winter and S. Nowak, Phys. Chem. Chem. Phys., 2017, 19, 4962-4966.

23 S. Wiemers-Meyer, S. Jeremias, M. Winter and S. Nowak, Electrochim. Acta, 2016, 222, 1267-1271.

24 J. Riches, L. Knutsen, E. Morrey and K. Grant, Fire Saf. J., 2002, 37, 287-301.

25 M. Grützke, V. Kraft, W. Weber, C. Wendt, A. Friesen, S. Klamor, M. Winter and S. Nowak, J. Supercrit. Fluids, 2014, 94, 216-222.

26 A. Friesen, C. Schultz, G. Brunklaus, U. Rodehorst, A. Wilken, J. Haetge, M. Winter and F. Schappacher, ECS Trans., 2015, 69, 89-99. 